\title{
In vivo imaging of early stages of rheumatoid arthritis by a5 $\beta 1$-integrin- targeted positron emission tomography
}

Johannes Notni ${ }^{1 * \dagger} \mathbb{D}$, Florian T. Gassert ${ }^{2+}$, Katja Steiger ${ }^{1,5}$, Peter Sommer ${ }^{2}$, Wilko Weichert ${ }^{1,5}$, Ernst J. Rummeny ${ }^{2}$, Markus Schwaiger ${ }^{3}$, Horst Kessler ${ }^{4}$, Reinhard Meier ${ }^{2}$ and Melanie A. Kimm²

\begin{abstract}
Background: Rheumatoid arthritis (RA) is one of the most common rheumatic diseases. Joint inflammation and pathological growth of joint cartilage cause swollen and painful joints, which severely diminishes the patients' life quality. There is no causal treatment. Symptomatic therapies should start as early as possible to take maximal effect. Hence, diagnostic procedures capable of detecting affected joints before the onset of clinical symptoms are highly desirable. We explored the value of PET imaging of integrin subtypes av $\beta 3$ and a5 $\beta 1$ for early detection of RA foci in collagen-induced arthritis (CIA) mouse models.
\end{abstract}

Results: Development of RA in CIA mice was monitored by paw scoring, and av $\beta 3$ - and a5 $\beta 1$-integrin expression was quantified by $\mu$ PET using ${ }^{68} \mathrm{Ga}$-Avebetrin and ${ }^{68} \mathrm{Ga}$-Aquibeprin. For consecutive sections of selected decalcified joints (knee, ankle), arthritic degeneration and integrin expression were assessed by MOVAT staining and $\beta 3 / a 5$ immunohistochemistry ( $\mathrm{HC}$ ), respectively. $\beta 3$ - and a5- $\mathrm{IHC}$ revealed elevated levels of both av $\beta 3$ - and a5 $\beta 1$-integrin in arthritic joints. Unlike av $\beta 3$, a5 $\beta 1$ is strongly expressed in the proliferating synovial lining layer, which suggests that its presence is directly related to RA development. For mice with advanced RA ( 6 weeks after CIA), PET signals for a5 $\beta 1$-integrin were substantially stronger ( $>300 \%$ of baseline) than that of av $\beta 3$-integrin $(<200 \%)$. A longitudinal PET follow-up revealed that the manifestation of clinical symptoms of RA is preceded by upregulation of a5 $\beta 1$ - but not of av $\beta 3$-integrin.

Conclusion: a5 $\beta 1$-integrin PET could add a new functional imaging aspect to the portfolio of RA diagnostics because it appears to be a sensitive biomarker for early RA development. We suggest a5 $\beta 1$-integrin PET as a valuable tool to achieve a higher precision for early diagnosis of RA, including initial staging, monitoring of the disease course, and drug treatment, and for planning of radiosynoviorthesis (RSO).

Keywords: Rheumatoid arthritis, Positron emission tomography, Integrins, Animal models, Collagen-induced arthritis, Immunohistochemistry

\section{Background}

Rheumatoid arthritis (RA) [1] is one of the most common rheumatic diseases and affects between 0.3 and $1.0 \%$ of adults worldwide, with 5-50 new cases per 100,000 being diagnosed each year. RA is an inflammatory autoimmune disorder primarily affecting joints and is associated with clinical symptoms typical for inflammation, such as warm,

\footnotetext{
* Correspondence: johannes.notni@tum.de

${ }^{\dagger} J$ ohannes Notni and Florian T. Gassert contributed equally to this work.

'Institute of Pathology, Technische Universität München, Trogerstr. 18, 81675

Munich, Germany

Full list of author information is available at the end of the article
}

swollen, and painful joints. RA is presumably caused by both genetic predisposition and environmental factors. In contrast to osteoarthritis (OA) which is a widespread, noninflammatory degenerative joint disease characterized by a loss of cartilage, the RA-inducing autoimmune attack causes an abnormal growth of fibrovascular tissue on the surface of the joint cartilage, called pannus, but also an inflammation of the synovial membrane which leads to overproduction of synovial fluid and a degeneration of the underlying bone. Currently, there is no causal treatment for RA. The common symptomatic therapies thus aim to 
reduce pain, inflammation, and progress of degeneration, in order to preserve the joint function and the life quality of patients as long as possible. The established long-term therapies rely on the systemic application of disease-modifying anti-rheumatic drugs (DMARDs), above all, the immunosuppressive agent methotrexate (a folate receptor inhibitor) [2], or biologicals, such as tumor necrosis factor $\alpha$ (TNF- $\alpha$ ) inhibitors [3-5]. Such therapies are most successful when the treatment is started early and aggressively [6], which in consequence, highlights the paramount importance of an early diagnosis.

In the course of the disease, patients who do not sufficiently respond to drug treatment may undergo a synovectomy, that is, the surgical removal of the synovial membrane which represents the main area of inflammatory processes and disease progression, typically resulting in a reduction of the symptoms of inflammation. However, a comparable effect can be achieved by the metabolic inactivation of the synovium by means of radiosynoviorthesis (RSO, also termed radiosynovectomy), a non-invasive treatment of singular rheumatic joints. RSO as well as surgery can also be used early on to avoid the adverse effects of systemic medication. For RSO, formulations containing $\beta^{-}$-emitting radionuclides with intermediate half-lives and high, intermediate, or low $\beta^{-}$energy, namely ${ }^{90} \mathrm{Y}\left(t_{1 / 2}=2.7\right.$ days, $\left.E_{\beta}{ }^{\max }=2.26 \mathrm{MeV}\right),{ }^{186} \operatorname{Re}\left(t_{1 / 2}=3.7 \mathrm{~d}, E_{\beta}{ }^{\max }=890\right.$ $\mathrm{keV})$, or ${ }^{169} \operatorname{Er}\left(t_{1 / 2}=9.5 \mathrm{~d}, E_{\beta}{ }^{\max }=340 \mathrm{keV}\right)$ are injected directly into the joint cavity of large (knee), medium-sized (shoulder, wrist, ankle), or small (finger, toe) joints, respectively. The strictly local $\beta^{-}$irradiation inactivates the inflammatory cells (osteoblasts and fibroblasts), whereupon the inflammation stops and disease progression ceases. Like DMARDs, the RSO is most successful when it is started as early as possible, ideally at a pre-symptomatic stage when the cellular signaling leading to inflammatory processes has just begun, but joints are still fully functional, i.e., degeneration and pannus formation have not started yet. Since RSO requires the identification of affected joints, planning of RSO treatment could be enhanced by the sensitive and selective functional imaging of biomarkers that are associated with the early development stages of RA in joints.

The 1987 Traditional Diagnostic Criteria of the American College of Rheumatology (ACR) for the Diagnosis of RA and the 2010 ACR-EULAR classification criteria for rheumatoid arthritis are mainly based on the clinical signs of RA and are widely recognized as the standard classification tree format with the highest sensitivity, specificity, predictive value, and accuracy [7-9]. This scheme is frequently supported by imaging techniques, such as radiography, ultrasound, and magnetic resonance imaging (MRI) $[10,11]$. The latter facilitates an accurate diagnosis of early RA $[12,13]$, because it can be applied to confirm the presence of subclinical synovitis [14], to discover RA in clinical remission when there is no sign of swelling or tender joints [15] and to detect bone erosion with a higher sensitivity than plain radiography [16]. Furthermore, although it is not specific for $\mathrm{RA}$, positron emission tomography (PET) with $\left[{ }^{18} \mathrm{~F}\right] 2$ fluoro-2-deoxy-D-glucose (FGD) can reveal arthritic lesions, because the advanced inflammatory processes are generally associated with an elevated energy conversion and, consequently, a higher glucose consumption. Indeed, arthritic joints could be quite convincingly delineated by wholebody FDG-PET [17], and FGD-PET has been employed for the assessment of therapy response [18].

However, the sensitivity and also the prognostic and predictive value of the established imaging modalities are limited in view of their relative inability to specifically detect pre-symptomatic stages of RA, i.e., where morphology and functionality of joints is not yet affected but the cellular processes ultimately leading to joint destruction have already begun to change. In contrast to the abovementioned imaging approaches which are primarily determined to detect abnormal morphology or strongly altered metabolic rates, functional imaging of dedicated cellular biomarkers for RA could lead to a paradigm shift in the diagnosis and treatment of RA, particularly for patients with an elevated hereditary risk. Identification and functional imaging of such biomarkers is therefore highly important.

Since synovial angiogenesis is a key process in RA development $[19,20]$, it has been suggested as a specific target for imaging of early RA. Angiogenesis in $\mathrm{RA}$ is triggered by proliferating mesenchymal synovial cells as a response to their elevated levels of energy and oxygen consumption upon pannus formation. As a result, the cellular receptors involved in the angiogenesis signaling pathways are upregulated, among them the integrin subtypes $\alpha v \beta 3$ and $\alpha 5 \beta 1$ which were found to be expressed in synovial endothelial cells and chondrocytes [21]. Hence, the radiolabelled derivatives of RGD peptides [22], which were successfully applied for $\alpha v \beta 3$-integrin targeted PET in the context of cancer and cardiovascular diseases [23, 24], have also been evaluated in terms of their utility for RA imaging, but did not provide a substantial added value over and above FDG-PET [25]. Yet, the value of $\alpha 5 \beta 1$-integrin imaging [26] has not been evaluated in this context, although multiple evidence indicates that this subtype is a more reliable biomarker for angiogenesis [27] and it is upregulated earlier than $\alpha v \beta 3$-integrin $[28,29]$. Therefore, we aimed to elucidate the potential of a recently introduced $\alpha 5 \beta 1$-integrin targeting PET probe, ${ }^{68} \mathrm{Ga}$-Aquibeprin [30, 31], for the selective imaging of early angiogenesis in an established animal model for RA [32]. 


\section{Materials and methods Mouse model}

Procedures involving laboratory mice and their care were conducted in conformity with institutional guidelines and with approval from the responsible local authorities. In total, $33 \mathrm{DBA} / 1 \mathrm{JRj}$ mice (7-8 weeks old) were obtained from Janvier Labs (France). Thirty mice were randomly categorized into 1 of 5 groups, each group comprising 6 mice. All five groups were subject to collagen-induced arthritis (CIA) according to a published standard protocol [32]. Briefly, DBA/1 mice were subcutaneously injected with $50 \mu \mathrm{g}$ bovine type II collagen (Chondrex Inc., Redmont, USA) emulsified with equal amounts of Freund's complete adjuvant (Sigma Aldrich, Taufkirchen, Germany) into the base of the tail. Fourteen days later, mice were administered a booster injection at the base of the tail with Freud's incomplete adjuvant (Sigma). The control group comprised 3 mice and was not subject to CIA. Groups 1 to 5 were subjected to PET imaging and MRI at time points of 1, 2, 3, 4, and 6 weeks after the beginning of RA development, respectively, and the control group after 6 weeks.

Paw scoring was done by monitoring paw swelling according to established standard criteria [32]. Briefly, each paw was measured for thickness, swelling, warmth, and redness and rated on a scale of 0-4. Score 0 was given when no evidence of erythema and swelling was observed, score 1 for erythema and mild swelling confined to the tarsals or ankle joint, score 2 for erythema and mild swelling extending from the ankle to the tarsals, score 3 for erythema and moderate swelling extending from the ankle to metatarsal joints, and score 4 for erythema and severe swelling encompassing the ankle, foot and digits, or ankylosis of the limb.

\section{MR imaging}

MR images were acquired on Agilent/General Electric MR901 small animal MRI system. Mice were anesthetized with isoflurane and had a tail vein catheter implanted. Mice were placed on the scanner bed on their side, with the hind limb pulled out and placed with the ankle centered over a $3 \mathrm{~cm}$ flexible surface receive coil (RAPID Biomedical, Germany), held in place with adhesive tape. A 2D multislice spoiled gradient recalled echo sequence was used, with 6 slices of $0.5-\mathrm{mm}$ slice thickness, TR $50 \mathrm{~ms}$, flip angle $30^{\circ}$, receive bandwidth $31.25 \mathrm{kHz}$, and 80 averages. Scan timing, field of view, and acquisition matrix varied slightly depending on anatomical positioning but were approximately TE $2.05 \mathrm{~ms}$, matrix size $128 \times 64$, and field of view $10 \mathrm{~mm} \times 5$ $\mathrm{mm}$. Image slices were oriented approximately parallel to the bones of the leg, perpendicular to the surface of the paw. Baseline pre-injection and post-contrast injection scans were acquired in one session, approximately every 7 min, without moving the animal.
The MRI findings were evaluated by a radiologist (RM) who is experienced in imaging of arthritis. For evaluation of synovitis, the EULAR-OMERACT RAMRI score was applied [33]. Synovitis was scored on a scale of $0-3$ in each joint, with a score of 0 representing no synovitis and scores of 1-3 representing mild, moderate, and severe synovitis, respectively. Synovitis was defined as an abnormal increase in signal intensity in the area of the synovium after Gadofluorine P injection $(100 \mu \mathrm{mol} /$ kg body weight, invivoContrast $\mathrm{GmbH}$, Berlin, Germany) and an expanded synovial membrane.

\section{PET}

$\mu$ PET imaging was performed as described before [30]. Briefly, DBA mice, weighting $20-25 \mathrm{~g}$ at the time of final use for experiments, received injections of the radiopharmaceuticals for PET via tail vein catheters under isoflurane anesthesia. In order to achieve injections with constant amounts of biologically active compound, $500 \mathrm{pmol}$ of the respective non-radioactive compound (Avebetrin or Aquibeprin, respectively) was added to each syringe and the actually injected total amount of cold mass calculated from syringe activities before and after injection as described previously [31]. For blockade, $30 \mathrm{nmol}$ of non-radioactive compound was added. For crossblockade, $30 \mathrm{nmol}$ of the respective other nonradioactive compound were added, i.e., Avebetrin to a ${ }^{68} \mathrm{Ga}$-Aquibeprin injection or Aquibeprin to a ${ }^{68} \mathrm{Ga}$-Avebetrin injection. Non-blockade animals received $18 \pm 3 \mathrm{MBq}$ with molar activities of $38 \pm 7$ $\mathrm{MBq} / \mathrm{nmol}$, and compound mass doses were $480 \pm$ $26 \mathrm{pmol}$. After injections, animals were allowed to wake up with access to food and water. PET was recorded under isoflurane anesthesia, $75 \mathrm{~min}$ p.i. for $20 \mathrm{~min}$, on a Siemens Inveon small-animal PET system. Generally, ${ }^{68} \mathrm{Ga}$-Aquibeprin and ${ }^{68} \mathrm{Ga}$-Avebetrin scans were recorded virtually simultaneously in relation to disease progress, that is, performed in the same order (first ${ }^{68} \mathrm{Ga}$-Aquibeprin then ${ }^{68} \mathrm{Ga}$-Avebetrin) on the same day with a 6-h interval allowing for virtually complete decay of the first injected radiopharmaceutical (overall residual activity calculated to approx. 2\%). Images were reconstructed as single frames using Siemens Inveon software, employing an ordered subset expectation maximum (OSEM) 3D algorithm without scatter and attenuation correction. All images shown are maximal intensity projections seen from dorsal, color scales ranging from 0 to $3 \%$ of injected dose per milliliter tissue.

Quantification of PET signals was performed by drawing spherical regions of interest (ROI) of standard volumes for knees $\left(23.5 \mathrm{~mm}^{3}\right)$ and ankles $\left(3.4 \mathrm{~mm}^{3}\right)$, centered to the respective voxels with 
maximal intensity. Paws were not analyzed because their small size and the resulting pronounced partial volume effect does not allow for reliable quantification of uptake. $\left[{ }^{68} \mathrm{Ga}\right.$ features a high positron energy $\left(\mathrm{E}_{\beta+, \max }=1.9 \mathrm{MeV}, \mathrm{E}_{\beta+, \text { avg }}=836 \mathrm{keV}\right)$, resulting in a high inherent degree of blurring [34] The mean traveling distance of positrons in tissue prior to annihilation is $3-4 \mathrm{~mm}$, which is exceeding the size of a mouse paw. Thus, the fraction of positrons leaving the paw tissue before local annihilation is considered too high to allow for a reliable quantification of the resulting PET signal, even more so because the stretched-out paws are surrounded by air and such positrons readily escape from the field-of-view of the scanner. Hence, despite being clearly recognizable in the images, any observed PET signal in paws is to be considered underestimated, which is however not relevant for prospective human application because of the much larger size of subjects.] ROI-based uptake values (\% injected dose per $\mathrm{mL}$ tissue) were analyzed on a per-cohort basis and are expressed as average values \pm standard deviation.

\section{Histopathology}

After the last PET scan, mice were sacrificed, extremities fixed in $4 \%$ PBS buffered formalin for 48 $\mathrm{h}$, decalcified in Osteosoft (Merck, Darmstadt, Germany) for 4 weeks according to the manufacturer's instructions, and embedded in paraffin. Sagittal sections of $4-\mu \mathrm{m}$ thickness were cut, and consecutive sections stained with Movat pentachrome staining was performed using a staining kit (Morphisto, Frankfurt am Main, Germany). For immunohistochemistry (IHC), serial sections were stained using a Bond RXm system (Leica, Wetzlar,
Germany, all reagents from Leica, unless otherwise indicated) with primary antibodies against $\alpha 5$ integrin (abcam, ab 108327, diluted 1:10.000) or $\beta 3$ integrin (Cell Signaling, 13166S, diluted 1:300). Briefly, slides were deparaffinized using deparaffinization solution, pretreated with Epitope retrieval solution 2 (corresponding to EDTA buffer $\mathrm{pH} 8$ ) for $30 \mathrm{~min}$. Antibody binding was detected with a polymer refine detection kit without post-primary reagent and visualized with DAB as a dark brown precipitate. Slides were scanned (Leica AT2, Leica, Wetzlar, Germany) and evaluated using Imagescope Software (Leica, Wetzlar, Germany).

\section{Results}

Study outline

In groups $(n=6)$ of collagen-induced arthritis (CIA) mice [32], the development of clinical RA symptoms was monitored by paw scoring (Fig. 1a). For the right hind paws, the extent of synovitis was additionally assessed by contrast-enhanced MRI and quantified according to the EULAR-OMERACT RAMRI scoring scheme (Fig. 1b). At the following day, two independent, subsequent PET scans were performed, utilizing the tracers ${ }^{68} \mathrm{Ga}$-Avebetrin for $\alpha \mathrm{v} \beta 3$-integrin and ${ }^{68} \mathrm{Ga}$ Aquibeprin for $\alpha 5 \beta 1$-integrin, respectively, and the uptakes in knee and ankle joints of the hind legs were quantified (Fig. 1c-f). Thereafter, the animals were sacrificed, selected joints were removed, decalcified, and embedded. Consecutive sections were subjected to MOVAT pentachrome staining for assessment of the extent of arthritic degeneration, as well as to $\beta 3$ - and $\alpha 5$-immunohistochemistry (IHC) for determination of the expression patterns of the target integrins.

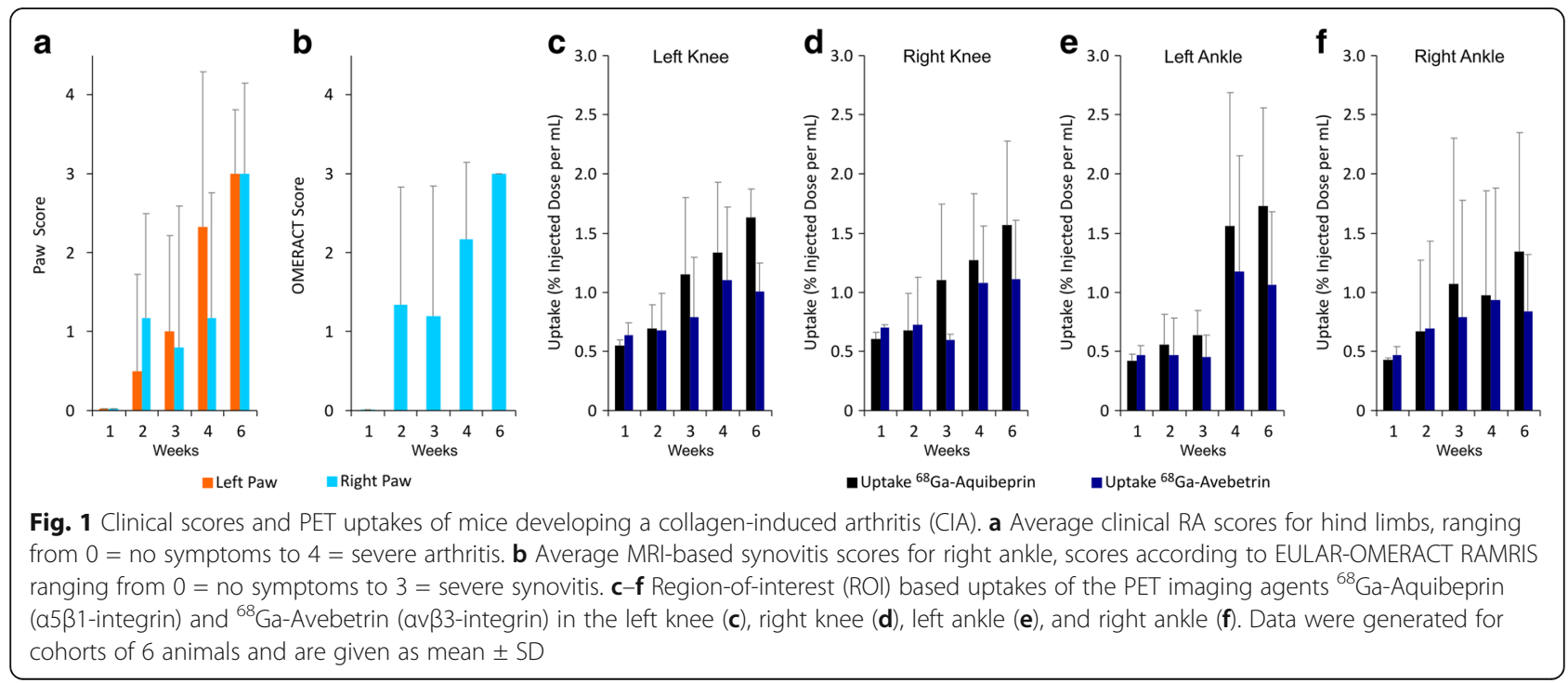




\section{PET imaging of RA-associated expression of av $\beta 3-$ and a5 $\beta 1$-integrin}

Figure 1 shows that the increase of the average clinical and MRI scores over time is accompanied with overall increasing integrin PET signals, which indicates that the integrin expression levels rise as the RA progresses. The consistently higher ${ }^{68} \mathrm{Ga}$-Aquibeprin uptakes suggest that the expression density of $\alpha 5 \beta 1$ integrin is higher, while a similar baseline uptake of both tracers (approx. $0.5 \% \mathrm{ID} / \mathrm{mL}$ in week 1 ) indicates that the stronger $\alpha 5 \beta 1$ signal is not related to a higher unspecific retention in the tissue.

In order to confirm the selectivity and specificity of both PET modalities for their respective integrin targets, we performed blockade and cross-blockade experiments for selected arthritic animals (Fig. 2). For both tracers, saturation of available receptors by co-injection of excess ligand resulted in a strong reduction of the PET signals in arthritic joints, proving that the observed signals are indeed caused by expression of the respective integrin and are not a result of unspecific accumulation mechanisms (Fig. 2a: control, 2.5; blockade, 0.5; Fig. 2c: control, 2.2; blockade, 0.5; uptakes given as average \% $\mathrm{ID} / \mathrm{mL}$ for joints marked with arrows). Additionally, independence of both signals was validated by saturation of the respective other receptor population by co-injection of an excess of the respective other ligands. Only slightly reduced signals for both combinations of imaging probe and blocking agent confirm the absence of cross-talk between the PET scans (Fig. 2b: control, 1.6; cross-blockade, 1.3; Fig. 2d: control, 2.0; blockade, 1.9; uptakes given as average $\% \mathrm{ID} / \mathrm{mL}$ for joints marked with arrows). Of note, the more pronounced reduction of the ${ }^{68} \mathrm{Ga}$-Aquibeprin signal is caused by a residual $\alpha 5 \beta 1$ integrin affinity of the co-injected Avebetrin (39 nM) [30]. This is too low to enable $\alpha 5 \beta 1$-integrin imaging with ${ }^{68} \mathrm{Ga}$ Avebetrin, but Avebetrin nevertheless competes to a small extent with ${ }^{68} \mathrm{Ga}$-Aquibeprin if administered in large amounts.

\section{IHC analysis of RA-associated a5 $\beta 1$-integrin levels}

The low PET background signal in the non-arthritic joints observed for both integrin tracers (see Fig. 3d, e, Fig. $1 c-f$ ) is in good agreement with the only focal weak IHC staining for the $\alpha 5$ and $\beta 3$ proteins in the respective regions (Fig. $3 \mathrm{~b}, \mathrm{c}$ ). Of note, $\alpha 5$-IHC is specific for $\alpha 5 \beta 1$ because $\alpha 5$ only dimerizes with $\beta 1$. In contrast, the $\beta 3$ specific antibody exhibits a cross-reactivity because $\beta 3$ also dimerizes with the $\alpha$ IIb chain whereby the platelet integrin $\alpha \operatorname{IIb} \beta 3$ is formed. The focal $\beta 3$ expression which is observed in the bone marrow (Fig. 3c) can be attributed to the presence of $\alpha \operatorname{IIb} \beta 3$-positive megakaryocytes and platelets, and thus, it does not indicate $\alpha v \beta 3$ integrin presence.

In contrast, the arthritic joints mostly show higher expression levels of $\alpha 5$-integrin than $\beta 3$, as illustrated by a representative example (Fig. 4). The reactive synoviocytes present with a strong expression of $\alpha 5$-integrin, whereas $\beta 3$-integrin is not identified within the synovial membrane. This is in agreement with the findings of other groups who reported that $\alpha 5$-integrin expression is increased in the synovial lining layer [21]. The advanced stages of RA show a moderate arthritis with mild

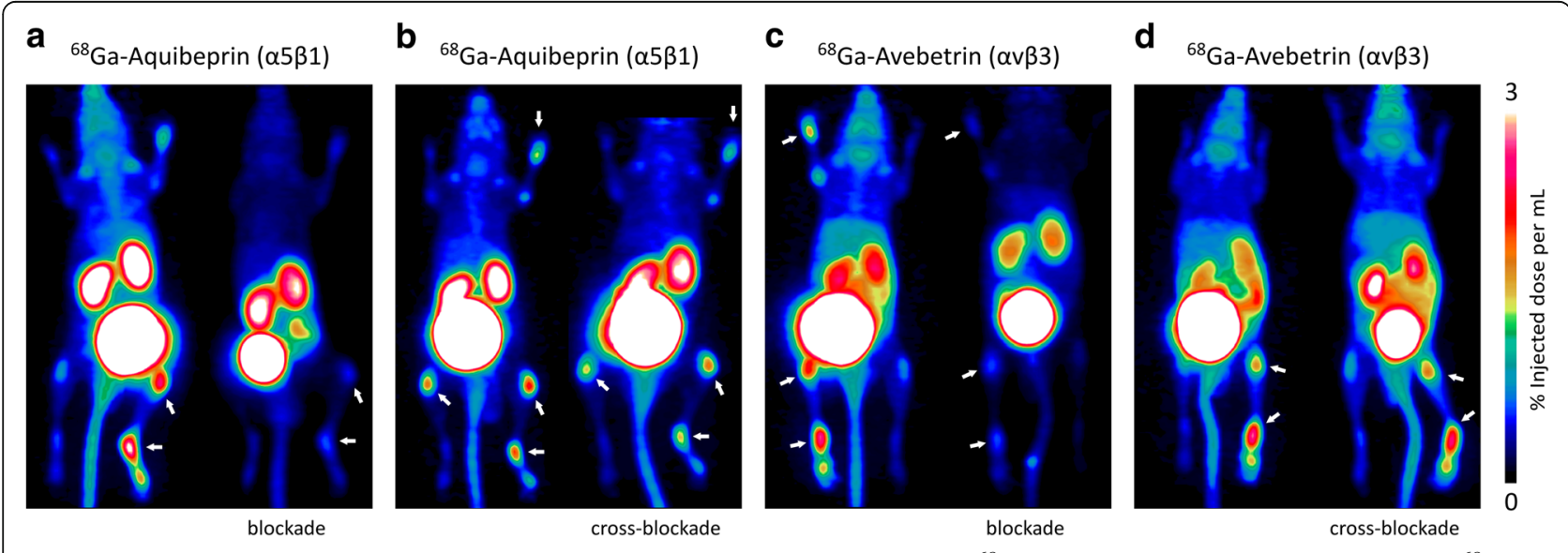

Fig. 2 Subtype-specific integrin PET imaging in arthritic mice. a, b a5 $\beta 1$ integrin PET using ${ }^{68}$ Ga-Aquibeprin. $\mathbf{c}$, d av $\beta 3$ integrin PET using ${ }^{68}$ GaAvebetrin. Each of the images $\mathbf{a}-\mathbf{d}$ shows two independent scans of the same mouse (different animals are shown in $\mathbf{a}-\mathbf{d}$ ). The arthritic joints are indicated by the arrows. The saturation of the receptor binding capacity by means of co-injection of a large dose ( $30 \mathrm{nmol}$ ) of unlabeled compound (blockade) resulted in a virtually complete reduction of the uptake in arthritic joints for both tracers, confirming the specificity of the PET imaging (a, c). The cross-blockade experiments confirmed the subtype-selectivity of a5 $\beta 1$ - and av $\beta 3$ integrin PET: The co-injection of 30 nmol Avebetrin did not substantially affect the a5 $\beta 1$ integrin imaging with ${ }^{68} \mathrm{Ga}$-Aquibeprin, while the minuscule reduction of the signal is likely related to a residual a5 $\beta 1$ activity $(39 \mathrm{nM})$ of Avebetrin (b). The co-injection of $30 \mathrm{nmol}$ Aquibeprin did not affect av 33 integrin imaging with ${ }^{68} \mathrm{Ga}$-Avebetrin $(\mathbf{d})$ 

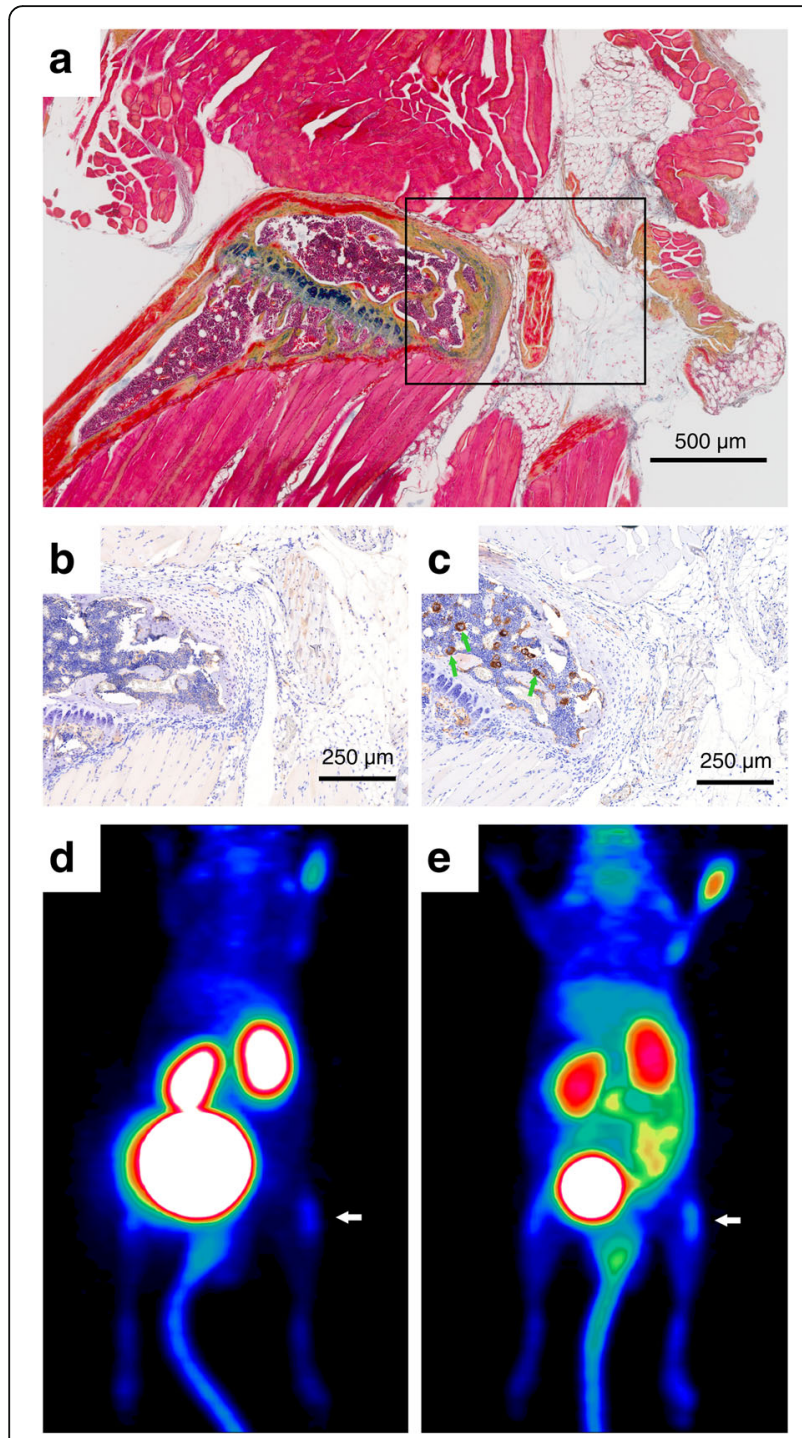

Fig. 3 Integrin expression in non-arthritic joints. a MOVAT pentachrome staining of the right knee of a CIA mouse from week 2. The immunohistochemistry staining of consecutive slices for integrin $a 5$ (b) and $\beta 3(\mathbf{c})$, (depicted area corresponds to the inset frame in $\mathbf{a})$. The same mouse was subjected to PET imaging of a5 $\beta 1$-integrin using 68Ga-Aquibeprin (d) and of av $\beta 3$-integrin using

${ }^{68} \mathrm{Ga}$-Avebetrin (e), wherein the white arrow indicates the knee subjected to staining $(\mathbf{a}, \mathbf{b}, \mathbf{c})$. No significant a5 expression was found (b), confirming the absence of a5 $\beta 1$. Focal expression of $\beta 3$ (c, green arrows) originates from the expression of the platelet integrin allb $\beta 3$ by megakaryocytes (platelet progenitor cells); no significant $\beta 3$ expression elsewhere confirms the absence of av $\beta 3$. Consistently, both tracers show a low uptake in a non-arthritic knee. Color coding of $\mu$ PET images $(\mathbf{d}, \mathbf{e})$ is similar to Fig. 2

cartilage and bone erosions as well as a hyperplastic synovitis associated with a strong $\alpha 5$-integrin expression (Fig. 4c-e), but still no $\beta 3$-integrin staining (Fig. $4 \mathrm{~g}-\mathrm{i}$ ) within the synoviocytes. Throughout the RA development, high 33 -integrin levels in macrophages and megakaryocytes were observed (Fig. 3c, Fig. 4i). The vascular endothelia within the vascular granulation tissue/pannus are also positive for $\alpha 5$-integrin (Fig. 4e), but only moderately express $\beta 3$-integrin (Fig. 4i).

\section{Discussion}

\section{Is a5 $\beta 1$-integrin a more specific biomarker for RA than} av $\beta 3$-integrin?

IHC (Fig. 4c-e) has shown that $\alpha 5 \beta 1$-integrin is predominantly found in the strongly proliferating synovial lining layer. This finding suggests that its expression is closely related to the specifically RA-related process of pannus formation and less so to a secondary process like angiogenesis. Hence, $\alpha 5 \beta 1$-integrin might evolve as a more specific biomarker for RA in joints than $\alpha v \beta 3$-integrin, whose upregulation is less specifically related to RA development but more so to the accompanying angiogenesis. Hence, we assume that $\alpha 5 \beta 1$-integrin-directed diagnostic procedures for RA, such as molecular imaging, are less susceptible to interference with other biochemical processes involving angiogenesis than $\alpha v \beta 3$-integrin-targeted approaches. At least, any RA-associated $\alpha 5 \beta 1$-integrin signals should be less related and/or superimposed by angiogenesis than those obtained for $\alpha v \beta 3$-integrin, and, thus, be more specific for RA.

\section{Value of a5 $\beta 1$-integrin vs. $\alpha v \beta 3$-integrin PET for prediction of RA progression}

Our data confirmed that the specific PET imaging of RA is feasible by targeting integrins $\alpha v \beta 3$ and $\alpha 5 \beta 1$, partly corroborating the earlier preclinical and clinical studies with radiolabelled $\alpha v \beta 3$-specific RGD peptides [25]. In this context, though, we recognize a substantial added value of $\alpha 5 \beta 1$-integrin PET. The evaluation of quantitative PET data reveals a somewhat lower baseline signal for $\alpha 5 \beta 1$ as compared to $\alpha v \beta 3$ (Fig. 5a; see also Fig. 3d, e) which might be beneficial for clinical application because a lower background usually goes hand in hand with a higher sensitivity and thus increases the likelihood to detect earlier stages of RA by PET.

More importantly, our PET data indicate that during the progression of RA, the $\alpha 5 \beta 1$-integrin is upregulated earlier than $\alpha v \beta 3$, raises steadily, and reaches higher levels than $\alpha v \beta 3$ at later stages of the disease, which becomes even more apparent if the intensity of the PET signals is normalized to the respective baselines (Fig. 5b). Of note, the average paw scores indicate a mild but stable clinical picture of RA in weeks 2 and 3, which advances to a more severe state in week 4 . This progress in week 4 is anticipated one week earlier by an $\alpha 5 \beta 1$-integrin expression, as the corresponding PET signal already increases in week 3 . In contrast, during weeks $2-4$, the $\alpha v \beta 3$-signal is only rising to the same extent as the paw scores. This observation strongly supports our hypothesis that $\alpha 5 \beta 1$-integrin PET might 

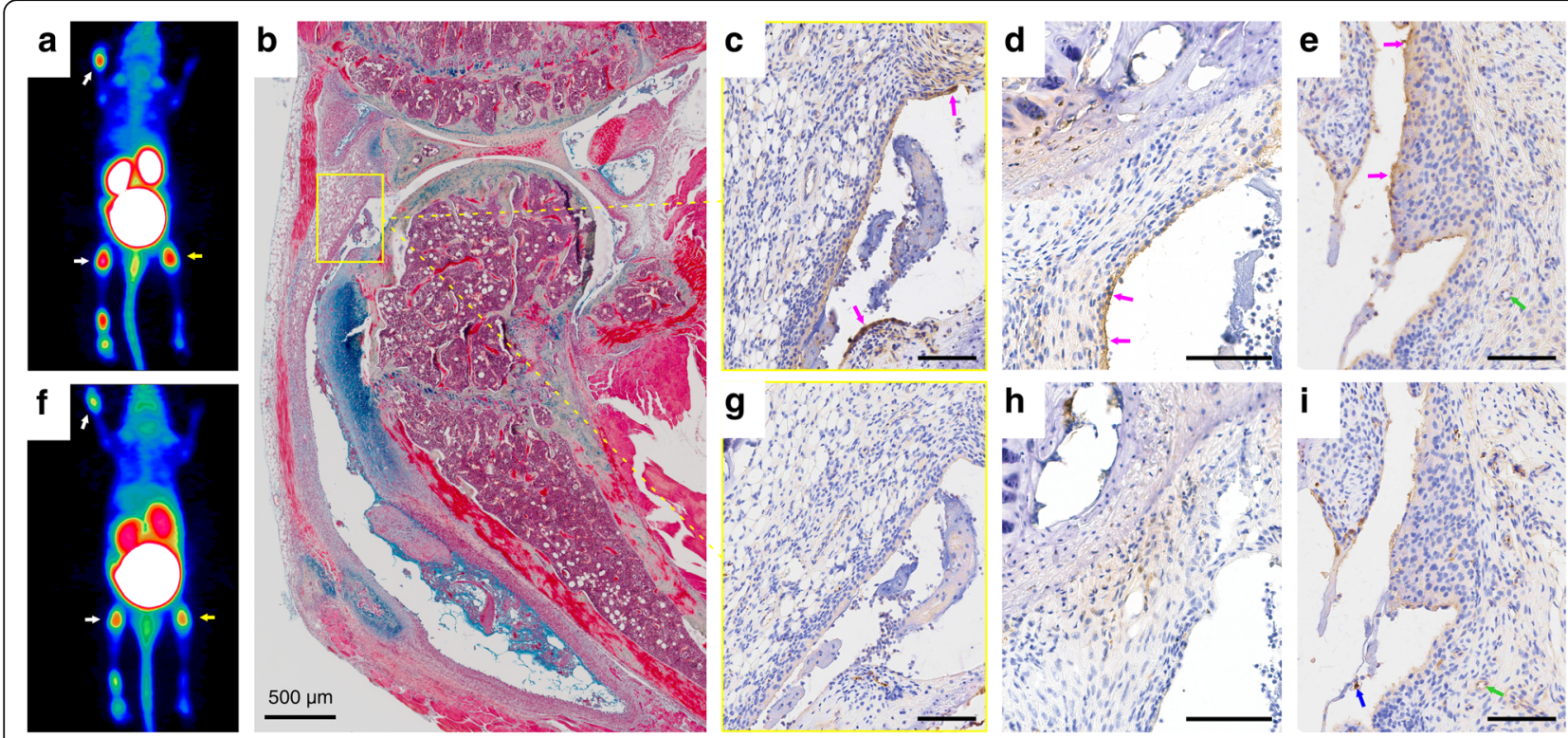

Fig. 4 Integrin expression in arthritic joints. a ${ }^{68} \mathrm{Ga}$-Aquibeprin (a531-integrin) $\mu$ PET for a DBA mouse from week 4. b MOVAT pentachrome staining of the right knee of the same animal used for $\mu \mathrm{PET}$ imaging $(\mathbf{a}, \mathbf{f}) . \mathbf{c}-\mathbf{e}$ a5-integrin immunohistochemistry (IHC). ${ }^{68} \mathrm{Ga}-\mathrm{Avebetrin}(\mathrm{av} \beta 3-$ integrin) $\mu P E T$ for the same animal shown in $\mathbf{a}$. $\mathbf{g}$-i $\beta 3$-integrin IHC. a5 and $\beta 3 \mathrm{IHC}$, respectively, are shown for consecutive slices of the right knee (c, $\mathbf{g}$; area shown corresponds to the frame in $\mathbf{b})$, left front paw $(\mathbf{d}, \mathbf{h})$, and left knee $(\mathbf{e}, \mathbf{i})$ of the same mouse used for PET (joints subjected to $I \mathrm{HC}$ are marked with arrows in $\mathbf{a}$ and $\mathbf{f}$ ). In many areas, the synovial membrane shows multifocal a5 expression (indicated by pink arrows in $\mathbf{c}$, $\mathbf{d}$, and $\mathbf{e}$ ) but virtually no presence of $\beta 3$. Vascular endothelia (indicated by green arrows) show only a slight expression of a5 (e) but a moderate level of $\beta 3$ (i). Furthermore, $\beta 3$ is expressed by macrophages (i, blue arrow). In the respective arthritic joints, overall stronger a5 expression gives rise to a more intense signal for ${ }^{68} \mathrm{Ga}$-Aquibeprin (a) as compared to ${ }^{68} \mathrm{Ga}$-Avebetrin (f). Unless otherwise noted, all scale bars indicate $100 \mu \mathrm{m}$. Color coding of $\mu$ PET images $(\mathbf{a}, \mathbf{f})$ is similar to Fig. 2

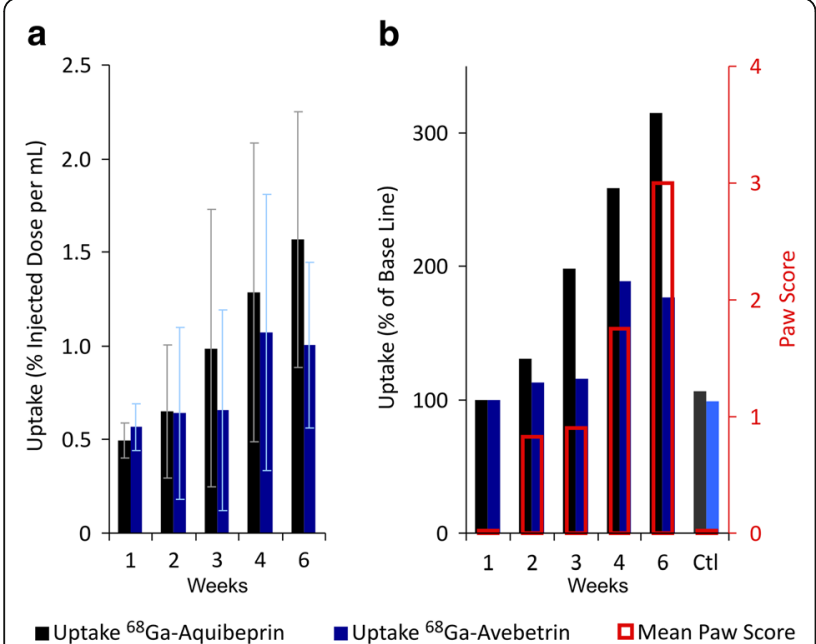

Fig. 5 Growth of integrin PET signals over time and correlation with clinical RA indicators. a Uptake of $68 \mathrm{Ga}$-Aquibeprin and ${ }^{68} \mathrm{Ga}-$ Avebetrin in the arthritic ankles and knees (mean $\pm \mathrm{SD}, n=24$ ), derived from PET data by an analysis of regions of interest (ROI). $\mathbf{b}$ Normalized uptakes (week $1=100 \%$ ) and mean paw scores at the time of imaging. Error bars omitted for clarity; for data with error bars, see Fig. 1a and Fig. 5a. Ctl: data for non-arthritic mice at week 6 (control, $n=12$ ) have a value for prediction of RA development. Furthermore, the $\alpha 5 \beta 1$ signal keeps rising with the paw scores during the progression of RA (week 4 to week 6) and ultimately reaches $>300 \%$ of the baseline. In contrast, the $\alpha v \beta 3$ signal is leveling below $200 \%$. These data substantiate that $\alpha 5 \beta 1$-integrin PET is more suitable for RA imaging. The specificity of PET for RA was furthermore confirmed by control animals without RA induction, which showed only the baseline uptake at the end point (Fig. 5b).

\section{Prospects of a5 $\beta 1$-integrin PET to improve clinical management of RA}

We have established that the $\alpha 5 \beta 1$-integrin PET enables the reliable localization of arthritic foci and furthermore anticipates RA progression. We conclude that this technique bears the potential to advance clinical management schemes of RA. Notably, the $\alpha 5 \beta 1$-integrin imaging adds a novel functional component to the diagnostic imaging portfolio, which could help to augment the established morphology-centered imaging methodology for RA and could provide additional information to medical practitioners suitable to achieve a higher precision in diagnostics, staging, and prognosis of RA.

Furthermore, we imagine an applicability for monitoring the therapy with DMARDs, which might be beneficial for 
patients because an early recognition of a lack of therapeutic effect might accelerate the decisions to terminate unsuccessful treatments and thus help to preserve the patients from the adverse effects of such medication. Furthermore, in light of the presumed predictive value of $\alpha 5 \beta 1$-integrin PET, it is reasonable to anticipate a substantial impact on RSO treatment planning, with the prospect of improving the life quality of RA patients who do not benefit from standard medication with DMARDs. Hence, we believe that $\alpha 5 \beta 1$-integrin PET will actually satisfy unmet clinical needs, demanding for immediate translation which is anticipated to result in routine clinical application.

\section{Conclusion}

We compared the time course and location of the expression of the integrin subtypes $\alpha 5 \beta 1$ and $\alpha v \beta 3$ in mouse models of rheumatoid arthritis (RA) by means of integrin-subtype specific PET imaging and immunohistochemistry. We found that $\alpha 5 \beta 1$ is predominantly located in the proliferating synovial lining layer, indicating that its expression might be closely related to a primary process of RA development in joints, namely, pannus formation. Hence, we assume that $\alpha 5 \beta 1$ is a more specific biomarker for RA development than $\alpha v \beta 3$ whose expression is merely related to a secondary process, angiogenesis. Consequently, we found that during the progress of RA, the signals of $\alpha 5 \beta 1$-integrin targeted PET are rising earlier and stronger than the respective $\alpha v \beta 3$-integrin PET signals. If the results of this study in murine models finally translate successfully to humans, $\alpha 5 \beta 1$-integrin PET could indeed offer the possibility for an early diagnosis of RA, possibly even before the manifestation of clinical symptoms. Since all RA treatment regimes benefit from an early start of therapy, $\alpha 5 \beta 1$ integrin PET might substantially improve the clinical management of RA and furthermore enhance the therapeutic outcome, particularly for patients with a hereditary risk.

\section{Acknowledgements \\ The authors thank Sybille Reder, Markus Mittelhäuser, and Marco Lehmann for assistance with animal PET, and Olga Seelbach and Marion Mielke for histological and immunohistochemical workup.}

\section{Authors' contributions}

JN, FTG, KS, PS, MAK, and RM conceived and designed the experiment. JN, FTG, KS, PS, and MAK performed the experiments. JN, FTG, KS, and MAK analyzed the data. JN, FTG, KS, and MAK wrote the original manuscript. JN, FTG, KS, PS, WW, EJR, MS, HK, MAK, and RM edited and revised the manuscript. All authors approved the final version of the manuscript.

\section{Funding}

This study was funded by the Deutsche Forschungsgemeinschaft (grants \#NO822/4-1, \#ME3718/2-1, \#ME3718/5-1, and SFB 824, projects A10 and Z2).

\section{Availability of data and materials}

The datasets used and/or analyzed during the current study are available from the corresponding author on reasonable request.
Ethics approval and consent to participate

Procedures involving laboratory mice and their care were conducted in conformity with institutional guidelines and with approval from the responsible local authorities.

\section{Consent for publication}

Not applicable.

\section{Competing interests}

The authors declare that they have no competing interests.

\section{Author details}

${ }^{1}$ Institute of Pathology, Technische Universität München, Trogerstr. 18, 81675 Munich, Germany. ${ }^{2}$ Department of Diagnostic and Interventional Radiology, Technische Universität München, Munich, Germany. ${ }^{3}$ Department of Nuclear Medicine, Technische Universität München, Munich, Germany. ${ }^{4}$ Institute for Advanced Study, Department of Chemistry, Technische Universität München, Garching, Germany. ${ }^{5}$ German Cancer Consortium (DKTK), Partner Site Munich, Munich, Germany.

Received: 15 May 2019 Accepted: 18 July 2019

Published online: 09 September 2019

References

1. Chaudhari K, Rizvi S, Syed BA. Rheumatoid arthritis: current and future trends. Nat Rev Drug Discov. 2016;15:305-6.

2. Lipsky PE, et al. Infliximab and methotrexate in the treatment of rheumatoid arthritis. N Engl J Med. 2000;343:1594-602.

3. Maini RN, et al. Therapeutic efficacy of multiple intravenous infusions of antitumor necrosis factor alpha monoclonal antibody combined with low-dose weekly methotrexate in rheumatoid arthritis. Arthritis Rheum. 1998;41:1552-63.

4. Feldmann M, Maini RN. Anti-TNF alpha therapy of rheumatoid arthritis: what have we learned? Annu Rev Immunol. 2001;19:163-96.

5. Jones G, Nash P, Hall S. Advances in rheumatoid arthritis. Med J Aust. 2017; 206:221-4

6. Saag KG, Teng GG, Patkar NM, et al. American College of Rheumatology 2008 recommendations for the use of nonbiologic and biologic disease-modifying antirheumatic drugs in rheumatoid arthritis. Arthritis Rheum. 2017;59:762-84.

7. Arnett FC, Edworthy SM, Bloch DA, et al. The American Rheumatism Association 1987 revised criteria for the classification of rheumatoid arthritis. Arthritis Rheum. 1988:31:315-24.

8. Sommer OJ, Kladosek A, Weiler V, Czembirek H, Boeck M, Stiskal M. Rheumatoid arthritis: a practical guide to state-of-the-art imaging, image interpretation, and clinical implications. Radiographics. 2005;25:381-98.

9. Aletaha D, Neogi T, Silman AJ, et al. 2010 Rheumatoid Arthritis Classification Criteria. Arthritis Rheum. 2010;62:2569-81.

10. Rizzo C, Ceccarelli F, Gattamelata A, et al. Ultrasound in rheumatoid arthritis. Med Ultrason. 2013;15:199-208.

11. Mäkinen H, Kaarela K, Huhtala H, Hannonen PJ, Korpela M, Sokka T. Do the 2010 ACR/EULAR or ACR 1987 classification criteria predict erosive disease in early arthritis? Ann Rheum Dis. 2012;72:745-7.

12. Sugimoto H, Takeda A, Hyodo K. Early-stage rheumatoid arthritis: prospective study of the effectiveness of MR imaging for diagnosis. Radiology. 2000;216:569-75.

13. Sugimoto H, Takeda A, Masuyama J, Furuse M. Early-stage rheumatoid arthritis: diagnostic accuracy of MR imaging. Radiology. 1996;198:185-92.

14. McQueen FM. The use of MRI in early RA. Rheumatology. 2008;47:1597-9.

15. Brown AK, Quinn MA, Karim Z, et al. Presence of significant synovitis in rheumatoid arthritis patients with disease-modifying antirheumatic druginduced clinical remission: evidence from an imaging study may explain structural progression. Arthritis Rheum. 2006;54:3761-73.

16. Østergaard M, Dohn UM, Ejbjerg BJ, McQueen FM. Ultrasonography and magnetic resonance imaging in early rheumatoid arthritis: recent advances. Curr Rheumatol Rep. 2006;8:378-85.

17. Kubota K, Ito K, Morooka M, et al. FDG PET for rheumatoid arthritis: basic considerations and whole-body PET/CT. Ann N Y Acad Sci. 2011;1228:29-38.

18. Yamashita H, Kubota K, Mimori A. Clinical value of whole-body PET/CT in patients with active rheumatic diseases. Arthritis Res Ther. 2014;16:423.

19. Marrelli A, Cipriani P, Liakouli V, et al. Angiogenesis in rheumatoid arthritis: a disease specific process or a common response to chronic inflammation? Autoimmun Rev. 2011;10:595-8. 
20. Paleolog EM. Angiogenesis in rheumatoid arthritis. Arthritis Res. 2002;4:S81-90.

21. Lowin T, Straub RH. Integrins and their ligands in rheumatoid arthritis. Arthritis Res Ther. 2011;13:244.

22. Kapp TG, Rechenmacher F, Neubauer S, et al. A comprehensive evaluation of the activity and selectivity profile of ligands for RGD-binding integrins. Sci Rep. 2017;7:39805.

23. Aumailley M, Gurrath M, Müller G, et al. Arg-Gly-Asp constrained within cyclic pentapeptides - strong and selective inhibitors of cell-adhesion to vitronectin and laminin fragment-P1. FEBS Lett. 1991;291:50-4.

24. Schottelius M, Laufer B, Kessler H, Wester HJ. Ligands for mapping av 33 integrin expression in vivo. Acc Chem Res. 2009:42:969-80.

25. Zhu Z, Yin Y, Zheng $K$, et al. Evaluation of synovial angiogenesis in patients with rheumatoid arthritis using ${ }^{68} \mathrm{Ga}$-PRGD2 PET/CT: a prospective proof-ofconcept cohort study. Ann Rheum Dis. 2014;73:1269-72.

26. Neubauer $\mathrm{S}$, Rechenmacher $\mathrm{F}$, Beer AJ, et al. Selective imaging of the angiogenic relevant integrins $a 5 \beta 1$ and av $\beta 3$. Angew Chem Int Ed. 2013;52:11656-9.

27. Fassler $\mathrm{R}$, Meyer M. Consequences of lack of $\beta 1$ integrin gene expression in mice. Genes Dev. 1995;9:1896-908.

28. Tanjore H, Zeisberg EM, Gerami-Naini B, Kalluri R. $\beta 1$ integrin expression on endothelial cells is required for angiogenesis but not for vasculogenesis. Dev Dyn. 2007;237:75-82.

29. Atkinson SJ, Ellison TS, Steri V, Gould E, Robinson SD. Redefining the role(s) of endothelial avß3-integrin in angiogenesis. Biochem Soc Trans. 2014;42:1590-5.

30. Notni J, Steiger $\mathrm{K}$, Hoffmann F, et al. Complementary, Selective PET-imaging of integrin subtypes $a_{5} \beta_{1}$ and $a_{v} \beta_{3}$ using Ga-68-Aquibeprin and Ga-68Avebetrin. J Nucl Med. 2016:57:460-6.

31. Notni J, Steiger K, Hoffmann F, et al. Variation of specific activities of Ga-68-Aquibeprin and Ga-68-Avebetrin enables selective PET-imaging of different expression levels of integrins $\alpha_{5} \beta_{1}$ and $a_{v} \beta_{3}$. J Nucl Med. 2016;57:1618-24

32. Brand DD, Kary AL, Rosloniec EF. Collagen-induced arthritis. Nat Protoc. 2007;2:1269-75.

33. Ostergaard M, Edmonds J, McQueen F, et al. An introduction to the EULAR-OMERACT rheumatoid arthritis MRI reference image atlas. Ann Rheum Dis. 2005;64:i3-7.

34. Sánchez-Crespo A, Andreo P, Larsson SA. Positron flight in human tissues and its influence on PET image spatial resolution. Eur J Nucl Med Mol Imaging. 2004;31:44-51.

\section{Publisher's Note}

Springer Nature remains neutral with regard to jurisdictional claims in published maps and institutional affiliations.

\section{Submit your manuscript to a SpringerOpen ${ }^{\circ}$ journal and benefit from:}

- Convenient online submission

- Rigorous peer review

- Open access: articles freely available online

- High visibility within the field

- Retaining the copyright to your article

Submit your next manuscript at $\boldsymbol{\nabla}$ springeropen.com 